LAWRENCE LIVERMORE NAT IO N A L LABORATORY

\section{Effect of the grain boundary on the evolution of deformation in a bicrystal}

Alexander Ziegler, Geoffrey H. Campbell, Mukul Kumar, James S. Stolken

September 8, 2004

iib2004

Belfast, United Kingdom

July 26, 2004 through July 29, 2004 
This document was prepared as an account of work sponsored by an agency of the United States Government. Neither the United States Government nor the University of California nor any of their employees, makes any warranty, express or implied, or assumes any legal liability or responsibility for the accuracy, completeness, or usefulness of any information, apparatus, product, or process disclosed, or represents that its use would not infringe privately owned rights. Reference herein to any specific commercial product, process, or service by trade name, trademark, manufacturer, or otherwise, does not necessarily constitute or imply its endorsement, recommendation, or favoring by the United States Government or the University of California. The views and opinions of authors expressed herein do not necessarily state or reflect those of the United States Government or the University of California, and shall not be used for advertising or product endorsement purposes. 


\section{Effect of the grain boundary on the evolution of deformation in a bicrystal}

A. Ziegler ', G. H. Campbell', M. Kumar', and J. S. Stölken ${ }^{2}$

${ }^{1}$ Materials Science and Technology Division, Chemistry and Materials Science Directorate, Lawrence Livermore National Laboratory, Livermore, CA 94551, USA

${ }^{2}$ New Technologies Engineering Division, Enginering Directorate, Lawrence

Livermore National Laboratory, Livermore, CA 94551, USA

Submitted to Interface Science for the Proceedings of the IIB 2004 Conference, Belfast, N. Ireland, UK, July 26 - 29, 2004.

July, 2004

This work was performed under the auspices of the U.S. Department of Energy by the University of California, Lawrence Livermore National Laboratory under Contract W-7405Eng-48. 


\title{
Effect of a grain boundary on deformation evolution in a bicrystal
}

\author{
Alexander Ziegler ${ }^{1}$, Geoffrey H. Campbell ${ }^{1}$, Mukul Kumar ${ }^{1}$ and James S. Stölken ${ }^{2}$ \\ ${ }^{1}$ Material Science and Technology Division, Chemistry and Materials Science Directorate \\ ${ }^{2}$ New Technologies Engineering Division, Engineering Directorate \\ Lawrence Livermore National Laboratory, University of California, Livermore, CA 94551, USA
}

\begin{abstract}
The role of grain boundary constraint in strain localization and concomitant constitutive response was examined by performing a series of uniaxial compression tests on a tantalum bicrystal. Tantalum single crystals were diffusion bonded to form a (011) $90^{\circ}$ twist boundary that was compressed along the common [011] direction. The plastic deformation resulted in the creation of deformation bands away from the highly constraining grain boundary, resembling those bands known from single crystal plastic deformation. Near the grain boundary, such deformation band formation could not be detected. Instead a distinctive pattern of crystal lattice rotation was observed that filled a rather large volume (several millimeters in size) around the bicrystal grain boundary. The internal deformation band structure as well as the crystal lattice rotation pattern near the bicrystal grain boundary were characterized and found to give greater rates of work hardening in the neighborhood of the grain boundary.
\end{abstract}

\section{Introduction}

Grain boundaries (GB) play a role in the deformation of polycrystalline metals beyond the well-known effects of grain size through the Hall-Petch relation. The types of GB present in a polycrystal are now known to influence deformation. Recent results show that, given a constant grain size, an fcc metal with moderate stacking fault energy $(\mathrm{Cu})$ that has been thermomechanically treated to increase the fraction of $\sum 3, \sum 9, \sum 27$, etc., GB present in the microstructure by the mechanism of GB decomposition [1] and has randomized texture [2] shows an increased yield and flow stress as compared to a normally processed polycrystal [3].

The investigation of polycrystals provides much information on grain boundaries in a plastically deforming metal. Complimentary information can be obtained through the detailed characterization of a plastically deforming bicrystal. We have chosen a bicrystal of Ta to study based on previous experience with single crystal deformation studies [4-6]. In these studies, single crystals of Ta were compressed along the [011] crystal direction to various levels of strain and the deformation microstructure was characterized through a variety of techniques. The deformation in this direction is highly constrained by the orientation of the available slip systems. Only two Burgers vectors are available for slip on two sets of (110) planes each. The [011] compression axis appears to be stable in orientation; the slip kinematics do not lead to a rotation of the macroscopic crystal orientation with respect to the compression axis. The two most highly stressed and active Burgers vectors produce material flow that causes an initially cylindrical mechanical test specimen to become oval in cross section. This behavior led then to the choice of a bicrystal orientation to study. The loading axis would remain the same [011] for the two crystals, however they would now be joined along a grain boundary perpendicular to the loading axis with a mutual $90^{\circ}$ twist 
misorientation. Characterizing the behavior of this grain boundary in such a highly constrained condition was the goal of the work.

\section{Experimental}

Tantalum single crystals for this investigation were grown from high purity material at the Institute for Solid State Physics in Chernogolovka, Russia into $18 \mathrm{~mm}$ diameter cylinders oriented along [013]. The single crystal was oriented using Laue back-reflection x-ray diffraction to cut slices with [011] longitudinal axis. After polishing and lapping the faces flat and parallel by specially developed techniques [7], the bicrystal was assembled by parallel alignment of the [100] axes of one crystal with the [01 1 ] axis of the other crystal (Figure 1). The grain boundary was chosen to be a $90^{\circ}$ twist boundary, thus resulting in maximal disorientation [8] for a BCC lattice. When considered within the coincident site lattice description of grain boundary structure [9], this grain boundary is found to have an irrational $\sum$ value due to the $\sqrt{2}$ ratio of spacings along the [100] and [01 $\overline{1}]$ directions that align parallel in this orientation. This configuration provided for symmetrical loading and therefore an identical far-field stress state and response to compression deformation in both single crystals, even at large strains. Additionally, it allowed for a multi-axial stress state at the grain boundary to be solely a function of the grain boundary geometry and the applied uniaxial loading.

The bicrystal was diffusion bonded [10] at $1500^{\circ} \mathrm{C}$ under $1 \mathrm{MPa}$ for 8 hours in ultra-high vacuum (UHV). This temperature is only slightly above half the melting point of Ta, so little mass transport is expected, however it did serve to stick the crystals together. A further anneal at $2400^{\circ} \mathrm{C}$ in UHV for 10 hours was performed to remove residual porosity at the grain boundary, with the added benefit of removing some of the interstitial impurities [11]. The final cylinder specimen dimensions of $6 \mathrm{~mm}$ in diameter and $18.6 \mathrm{~mm}$ in height was cut from the bicrystal using electric spark discharge machining. The cylinder was finally turned to make the loading surfaces flat and parallel and a reference grid was placed in the surface of the specimen by scratching with a sharp needle.

An interesting feature about deformation along a $<011>$ axis in a BCC crystal lattice is the plain strain condition that persists to large strains. During compression along the [011] axis extensive plastic deformation occurs along [100], while only small changes ensue along [01 $\overline{1}$ ]. This planar deformation is attributed to slip along the directions of all potentially active $\{110\}$ and $\{112\}$ slip planes that are contained in a single $\{110\}$ plane. Thus, for applied loads along the [011] axis the active $\{110\}$ and $\{112\}$ slip planes occur in symmetric pairs, each pair containing both [111] and [ $\overline{1} 11]$ slip directions, and plastic deformation is limited to the $(01 \overline{1})$ plane, as illustrated in Figure 2.

The uniaxial compression deformation along [011] was performed using a screw driven test machine, deforming the specimen in $10 \%$ increments to a $30 \%$ total engineering strain at a strain rate of $10^{-4} \mathrm{~s}^{-1}$. The overall deformation behavior was then analyzed via tomographic volume reconstruction that allowed determination of the effective states of stress and strain over the crosssectional area, measured as a function of distance from the grain boundary. The tomographic routine used was a standard filtered back-projection routine that comes with the IDL programming language. A total of 36 images of the object acquired at equally spaced $5^{\circ}$ rotations about the 
compression axis were used to reconstruct the volume. The grain boundary profile was analyzed and finally post-test sectioning of the sample allowed characterization using electron microscopies.

Optical microscopy, scanning and transmission electron microscopies were used to examine the microstructure and the mechanisms that are active during plastic deformation. Electron backscattered diffraction was used to explore variations in the local lattice rotations. Individual lattice orientation measurements were performed at discrete points on the sample, which were subsequently used to construct lattice rotation maps of the investigated area.

\section{Results and Discussion}

Photographs of the deformed specimen are presented in Fig. 3, showing the two-fold symmetry developed in the shape of the bicrystal as the deformation increases from $10 \%$ to $30 \%$ engineering strain. The top and bottom crystals deform in accord with Ta-single crystal deformation experiments [4-6], whereby the initially circular sample diameter evolves into an ellipse (Figure 2). This result is also in agreement with drawn BCC polycrystalline $<011>$ textured wires that have demonstrated that initially cylindrical specimen can adopt an ovoid shape within the (011) plane [12].

When compressing along the [011] axis the crystals tend to respond with deformation almost entirely in the $(01 \overline{1})$ plane and predominantly the [100] direction by lattice rotation about the $[01 \overline{1}]$ axis. However, as seen in Figure 3, the $90^{\circ}$ twist GB clearly disturbs this deformation behavior. Neither crystal conforms to the single crystal response to compression loading in the vicinity of the GB. From the cross-sections presented in Figure 4 it becomes obvious that the GB is highly constrained; the initially flat GB evolved into a saddle-shaped surface in response to the constraint.

The GB can also be detected in the plots of the effective states of stress and strain over the cross-sectional area as a function of distance along the compression axis, presented in Figure 5a-c. The deformation dependence of the cross-sectional area (Fig. 5a) shows how the constrained GB allows for a small degree of lateral deformation by adjusting its absolute cross-sectional area in response to sample deformation. The area increases from initially $29 \mathrm{~mm}^{2}$ to approximately 38.5 $\mathrm{mm}^{2}$ at $30 \%$ strain. Figure $5 \mathrm{~b}$ shows the corresponding deformation dependence of the areal strain along the compression axis and the extensive strain gradient caused by the grain boundary. The downturn of the curves at both ends of the compression axis in both graphs is an end-effect that is due to the lateral friction forces acting between the sample and the testing machine plattens. The deformation dependence of the effective stress state along the compression axis shown in Fig. 5c, shows how the grain boundary region is stronger. Its constraint and the multi-axial stress state lead to increased work hardening.

It is instructive to compare the lattice rotation maps of the bicrystal with observations made on single crystals. The lattice rotation map of a Ta-single crystal exhibits a particular pattern of alternating lattice rotation throughout the entire sample. This pattern, when viewed at larger magnification, reveals a network of lattice rotation bands that comprise most of the crystal lattice rotation, while the areas in between those bands do not show evidence of lattice rotation activity. It 
is important to note, that these lattice rotation bands do not coincide with the traces of the $\{110\}$ or $\{112\}$ slip planes, furthermore, their orientation changes as strain progresses [6].

TEM images of the deformation bands, seen in the same orientation as in the lower crystal, are presented in Fig. 6. These images show that the deformation bands consist of two, almost parallel walls of high dislocation density, in between which more dislocations are accumulated. The analysis of a diffraction pattern of such a deformation band and its surrounding area (Fig 6b) reveals that the lattice inside the deformation band is rotated by approximately $5^{\circ}$. This level of rotation is consistent with observations from EBSD maps of these same regions.

Very similar lattice rotation bands and the alternating pattern can be observed to occur in the bicrystal, although they form away from the constrained grain boundary region, Fig. 7. The pattern forms broad areas of gradual and continuous lattice rotation that follows the macroscopic features and shades already observed in the optical image, Fig. 5. Since the lattice rotation in both crystals occurs about the [01 $\overline{1}$ ] axis, the EBSD map allows distinguishing to what degree and in what direction the lattice is rotated away from its original orientation. Thus, at the center of the sample the lattice very near the constrained grain boundary rotates only a few degrees. Alternatively, when moving away from the center towards the outer sample boundary and along the axis of compression [011], lattice rotation increases to about $20^{\circ}$ on both sides of the grain boundary; forming different patterns respectively.

\section{Conclusions}

A highly constraining $90^{\circ}$ twist-grain boundary in a Ta-bicrystal was found to clearly disrupt the homogeneous deformation behavior of both single crystals. The overall response of the bicrystal to plastic deformation, ranging from $10 \%$ to $30 \%$ total engineering strain did show similar features to the single crystal experiment only in the non-constrained regions of the sample, away from the grain boundary, i.e., planar double-slip deformation predominantly in the $(0 \overline{1} 1)$ plane and an elliptical cross-sectional profile. However, close to the grain boundary the crosssectional profile did not deform accordingly and remained highly constrained exhibiting only limited lateral deformation. The grain boundary adjusted to accommodate the deformation of the adjacent crystals by taking on a saddle-shaped profile.

A large strain gradient could be measured near the grain boundary and the boundary itself presented a stronger area of the sample. This was attributed to the constraint and the multi-axial stress state, causing increased hardening from multiple slip system activation. We anticipate that the size of this region would scale with the size of the bicrystal. Interpreting the behavior observed in the bicrystal in terms of its implications for polycrystal behavior is rather speculative at this point. One can imagine a grain in a polycrystal, surrounded by other grains that constrain its deformation such that multiple slip systems are always activated, leading to increased hardening. However, a condition in which the neighboring grains were less constraining, such as in a textured material where the grains are more nearly in the same orientation, would lead to differing slip system activity and a different rate of work hardening.

The examination using EBSD resulted in lattice rotational patterns, resembling observations made in the corresponding single crystal deformation experiments. Here, lattice rotation of up to $5^{\circ}$ was limited only to the deformation bands. However, the constrained grain boundary affected 
the distribution of such patterns. A large volume, spanning several millimeters along and across the grain boundary, with evidence of lattice rotation was observed on both sides of the grain boundary. Unlike the discrete lattice rotation limited to the deformation bands, the material near the grain boundary exhibited broad areas of continuous lattice rotation.

\section{Acknowledgements}

This work performed under the auspices of the Office of Basic Energy Sciences, U.S. Department of Energy by University of California, Lawrence Livermore National Laboratory under Contract W-7405-Eng-48.

\section{References}

1. $\quad$ M. Kumar, A. J. Schwartz and W. E. King, Acta Mater. 50, 2599 (2002).

2. M. Kumar, A. J. Schwartz and W. E. King, Mater. Sci. Eng. A 309 - 310, 78 (2001).

3. M. Kumar, K. J. Blobaum and J. S. Stolken, (unpublished results).

4. A. J. Schwartz, W. E. King, G. H. Campbell, J. S. Stolken, D. H. Lassila, S. Sun and B. L. Adams, in Fracture and Ductile vs. Brittle Behavior; Theory, Modeling \& Experiment, edited by G. E. Beltz, R. L. Blumberg-Selinger, M. P. Marder and K.-S. Kim (Materials Research Society, Pittsburgh, PA, 1999), p. 221

5. A. J. Schwartz, W. E. King, G. H. Campbell, J. S. Stölken, D. H. Lassila, S. Sun and B. L. Adams, ASME J. Eng. Mater. Tech. 121, 178 - 181 (1999).

6. A. J. Schwartz, J. S. Stolken, W. E. King and G. H. Campbell, Mater. Sci. Eng. A 317, 77 (2001).

7. W. L. Wien, G. H. Campbell and W. E. King, in Microstructural Science, edited by D. W. Stevens, E. A. Clark, D. C. Zipperian and E. D. Albrecht (ASM International, Materials Park, OH, 1996), p. 213

8. K. S. Havner, Finite plastic deformation of crystalline solids (Cambridge University Press, New York, NY, 1992).

9. W. Bollmann, Crystal Defects and Crystalline Interfaces (Springer - Verlag, Berlin, 1970).

10. W. E. King, G. H. Campbell, A. W. Coombs, G. W. Johnson, B. E. Kelly, T. C. Reitz, S. L. Stoner, W. L. Wien and D. M. Wilson, in Joining and Adhesion of Advanced Inorganic Materials, edited by A. H. Carim, D. S. Schwartz and R. S. Silberglitt (Materials Research Society, Pittsburgh, PA, 1993), p. 61

11. R. L. Smialek and T. E. Mitchell, Philos. Mag. 22, 1105 (1970).

12. W. F. Hosford Jr., Trans. Metall. Soc. AIME 230, 12 (1964). 


\section{Figure Captions}

Fig. 1 Bicrystal geometry with $90^{\circ}$ twist boundary and the [011] compression axis.

Fig. 2 Deformation behavior of a Ta-single crystal is controlled by the two Burgers vectors indicated in the drawing. The initial profile of the test specimen is circular and evolves into an oval due to the kinematics of the planar slip occurring in the $(01 \overline{1})$ plane.

Fig. 3 Plastically deformed Ta-bicrystal at 10, 20 and 30\% strain showing two-fold symmetry due to the constrained grain boundary. Images such as these were used for the tomographic reconstruction of the shape of the specimen.

Fig. 4 The deformation dependence of the cross-sectional area (a) and the effective states of areal strain (b) and stress (c) over the cross-sectional area are shown. Although the grain boundary is highly constrained, the cross section of the grain boundary shows evidence of lateral deformation and a large strain gradient. The higher strength of the grain boundary region after deformation is attributed to the change in the stress state due to the influence of the elastic anisotropy of the crystals across the boundary that activates additional slip systems and increases the hardening rate through forest hardening mechanisms.

Fig. 5 An overview of the entire deformed bicrystal is shown, as cross-sectioned along the [011] compression axis.

Fig. 6 a) TEM image of a deformation band showing that these bands consists of two, almost parallel walls of high dislocation density, in between which some more dislocations are accumulated.

b) Diffraction pattern of a deformation band and its surrounding area, revealing, that the lattice inside the deformation band is rotated by approximately $5^{\circ}$.

Fig. 7 An EBSD quality map is shown of the near grain boundary region on the center section of the mechanical test specimen. The bottom crystal has the [01 $\overline{1}]$ surface normal while the top crystal has the [100] surface normal. The deformation bands characteristic of the single crystal behavior can be seen on either side of the boundary at some distance from the boundary. This characteristic deformation microstructure is not observed in the crystal regions near the boundary. This subsection of the surface should be compared with Fig. 5 to locate it within the whole specimen. 

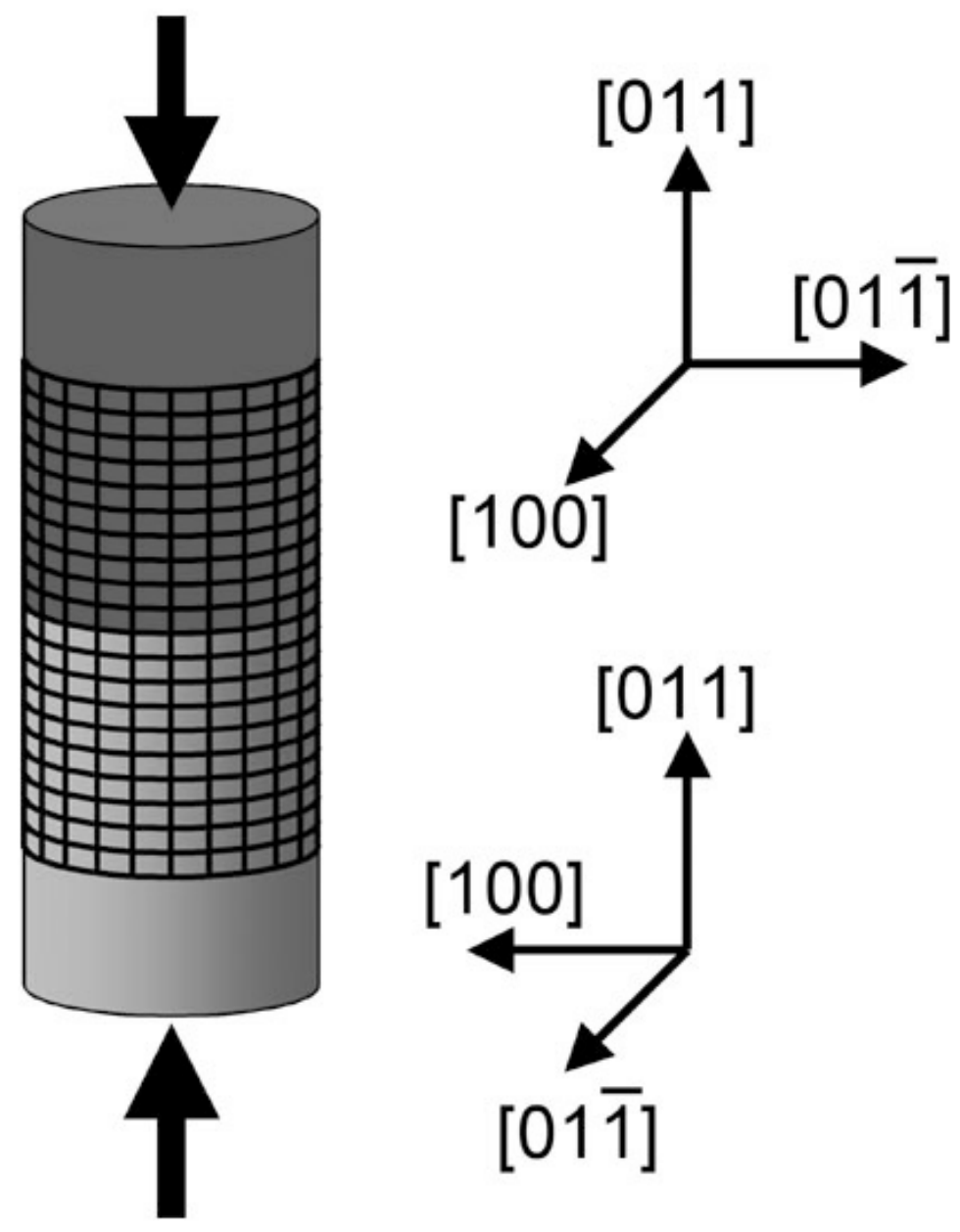

Figure 1 


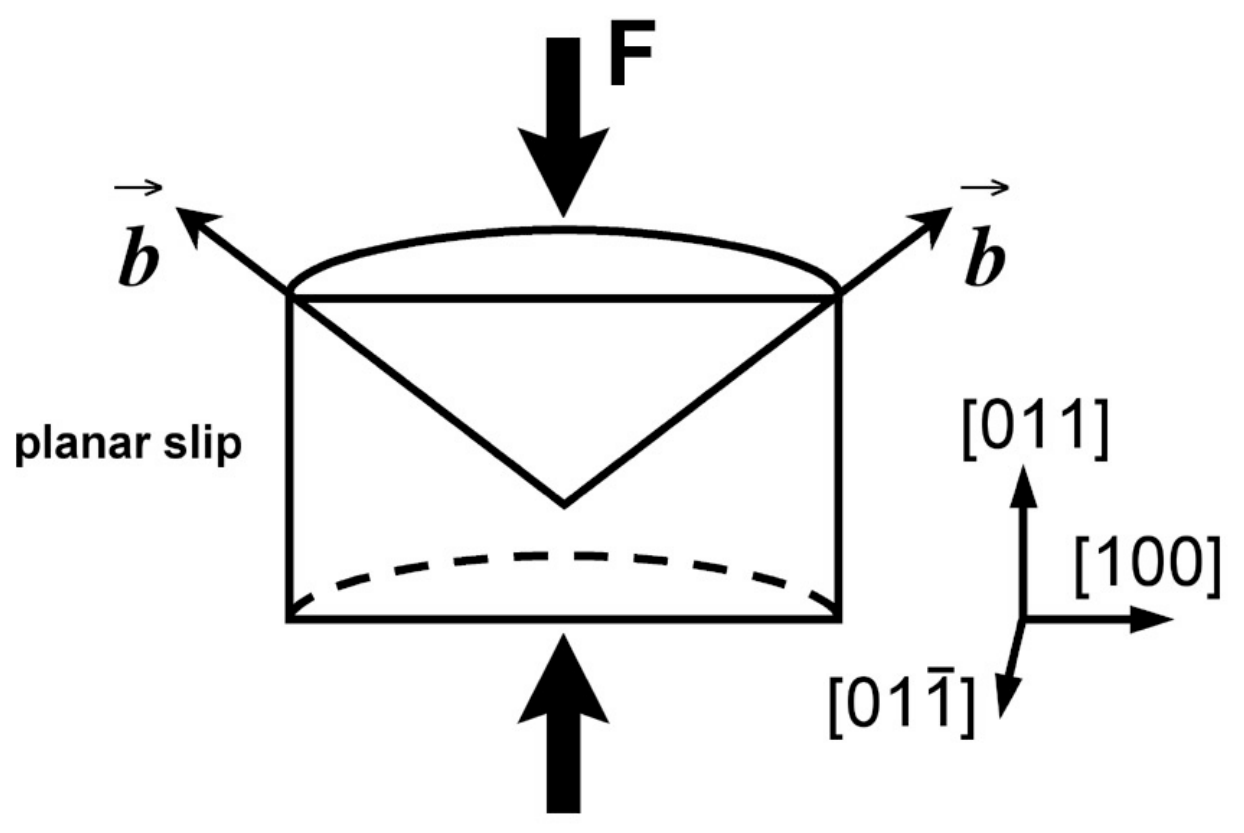

Figure 2 


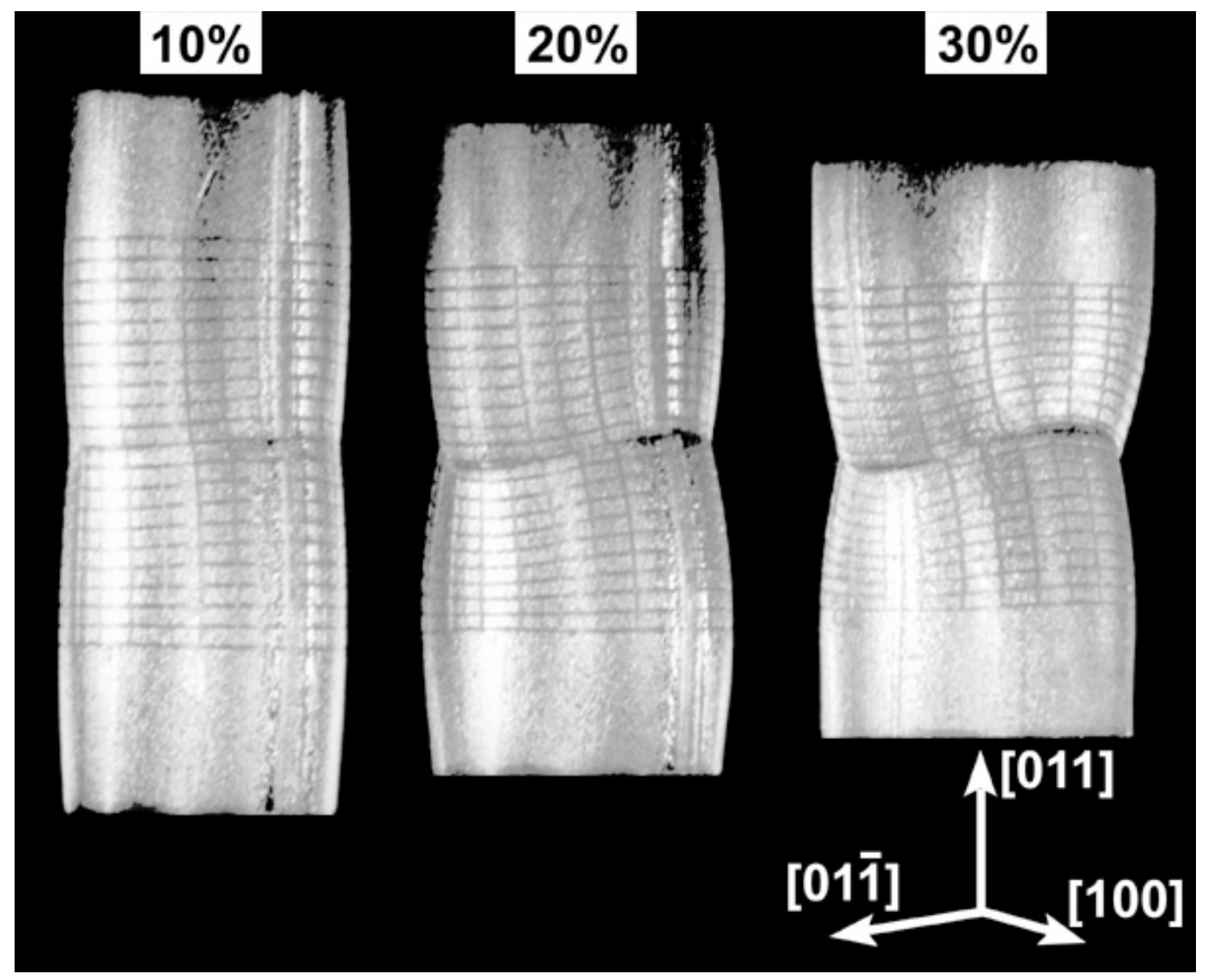

Figure 3 


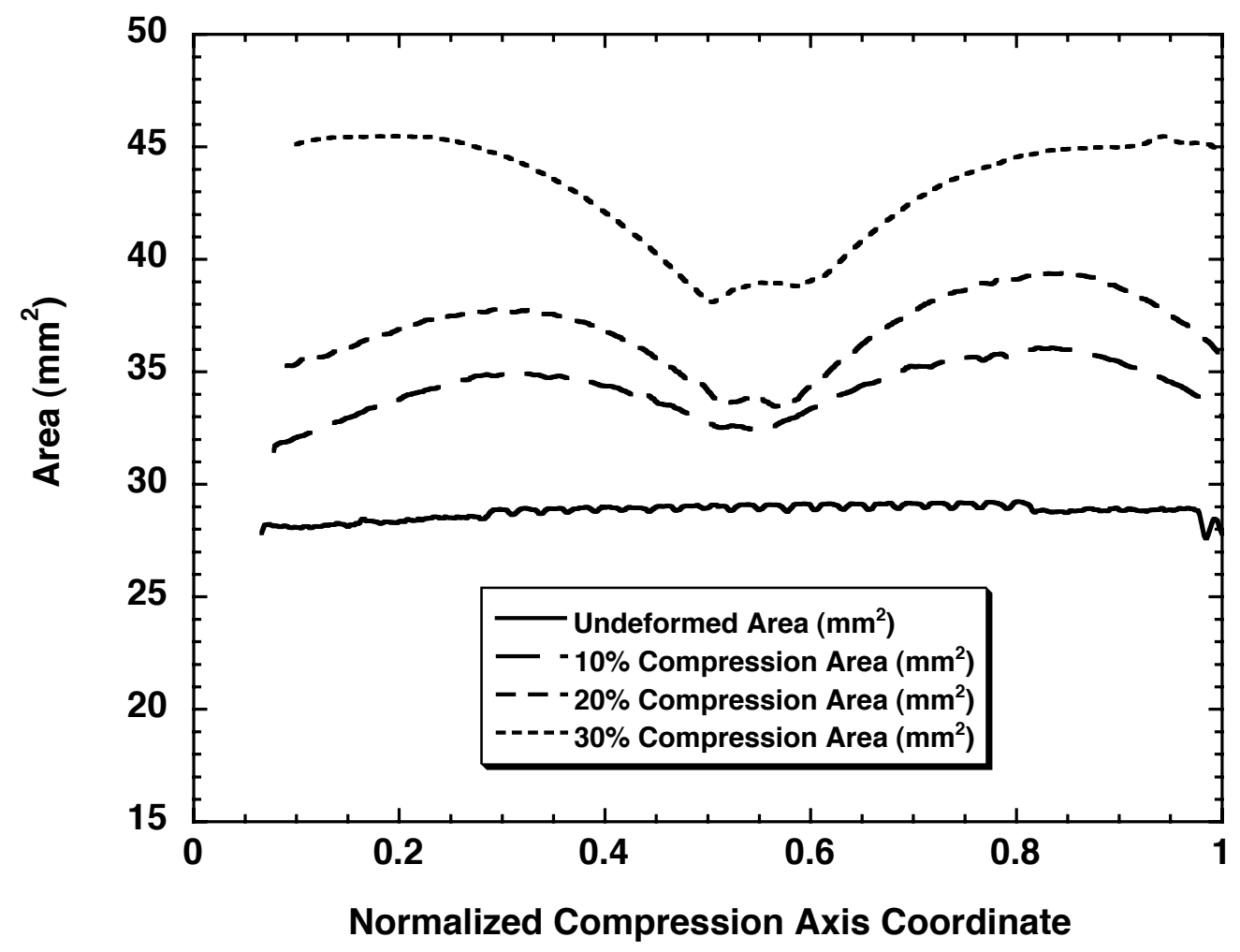

Figure 4a 


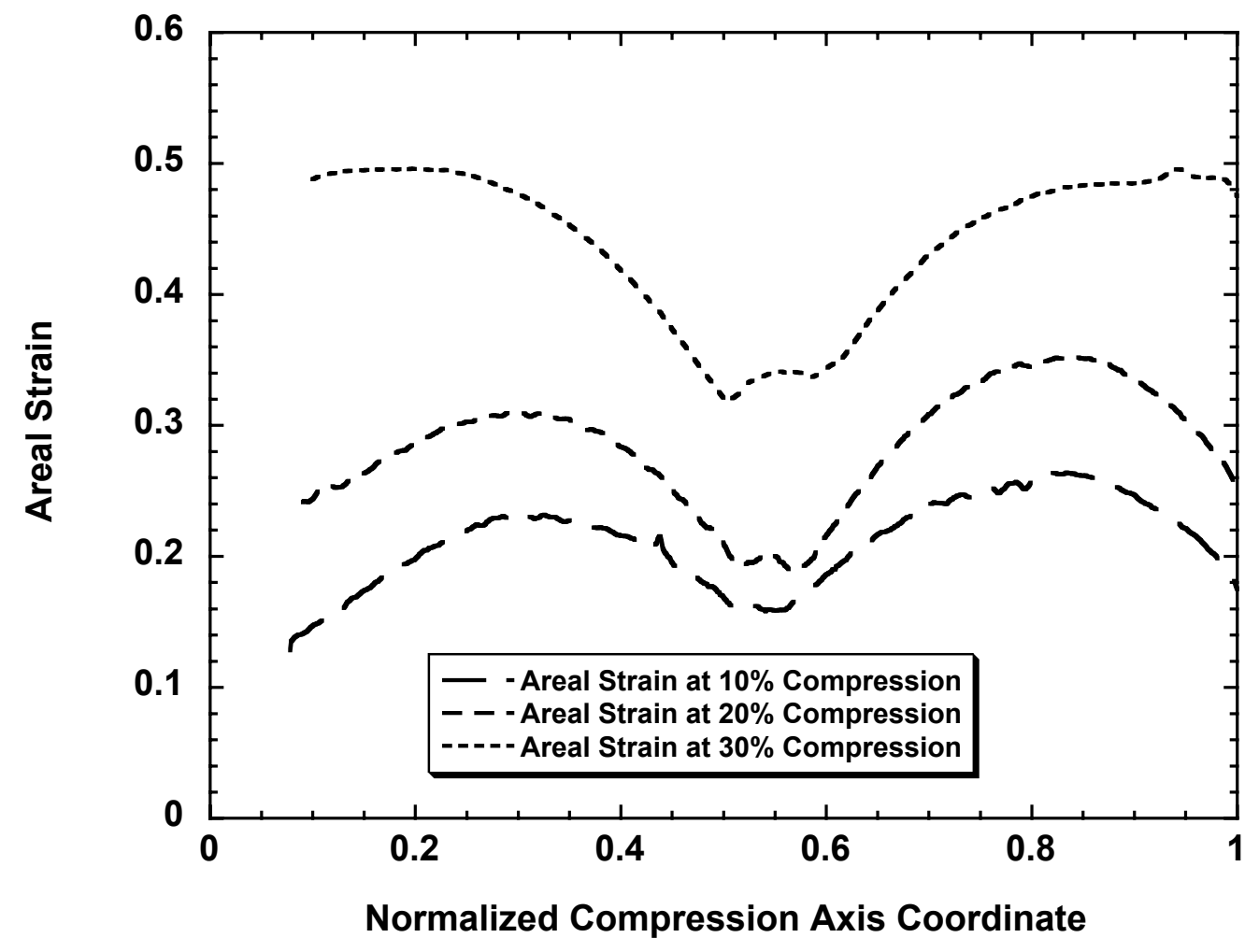

Figure 4b 


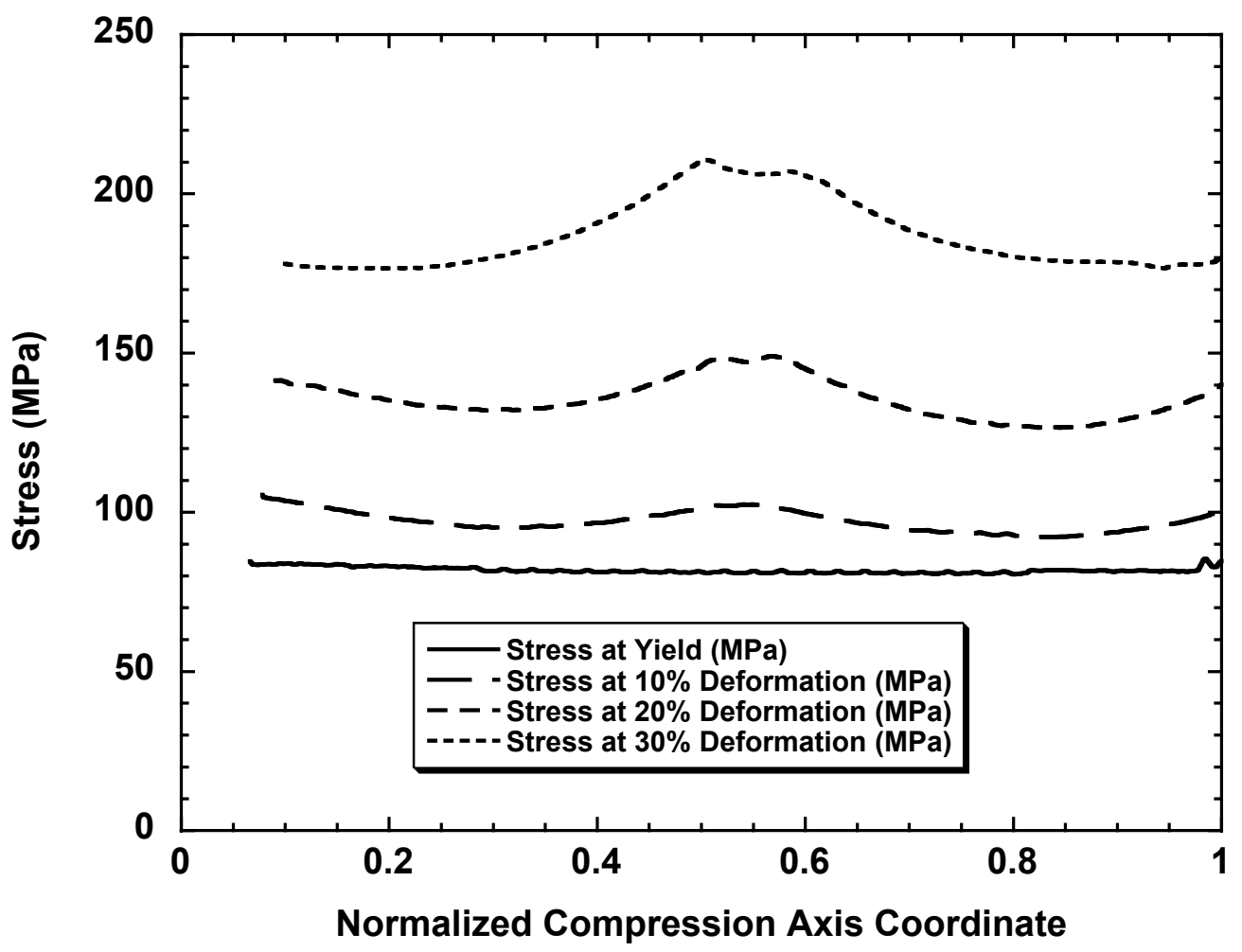

Figure 4c 

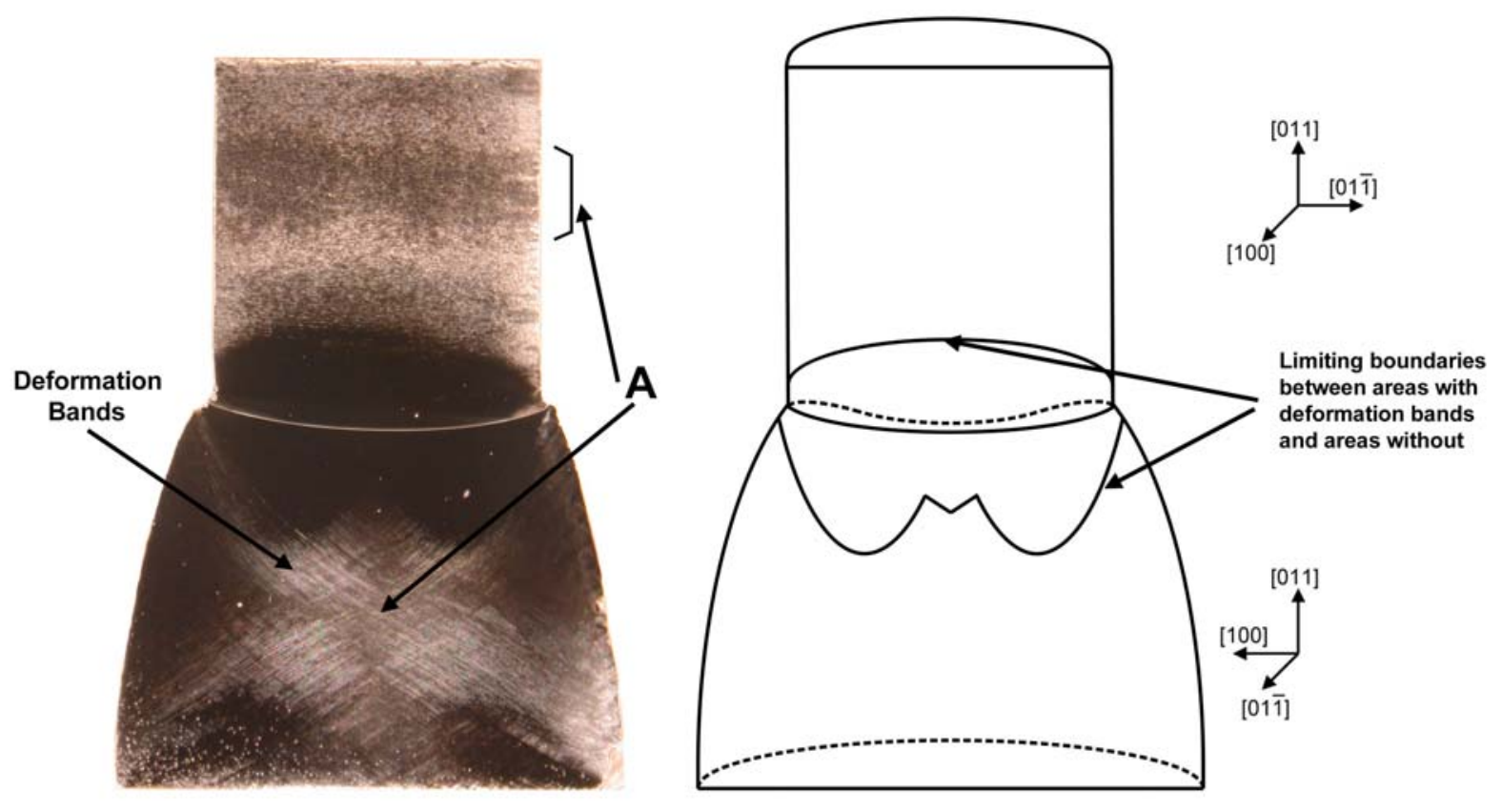

Figure 5 


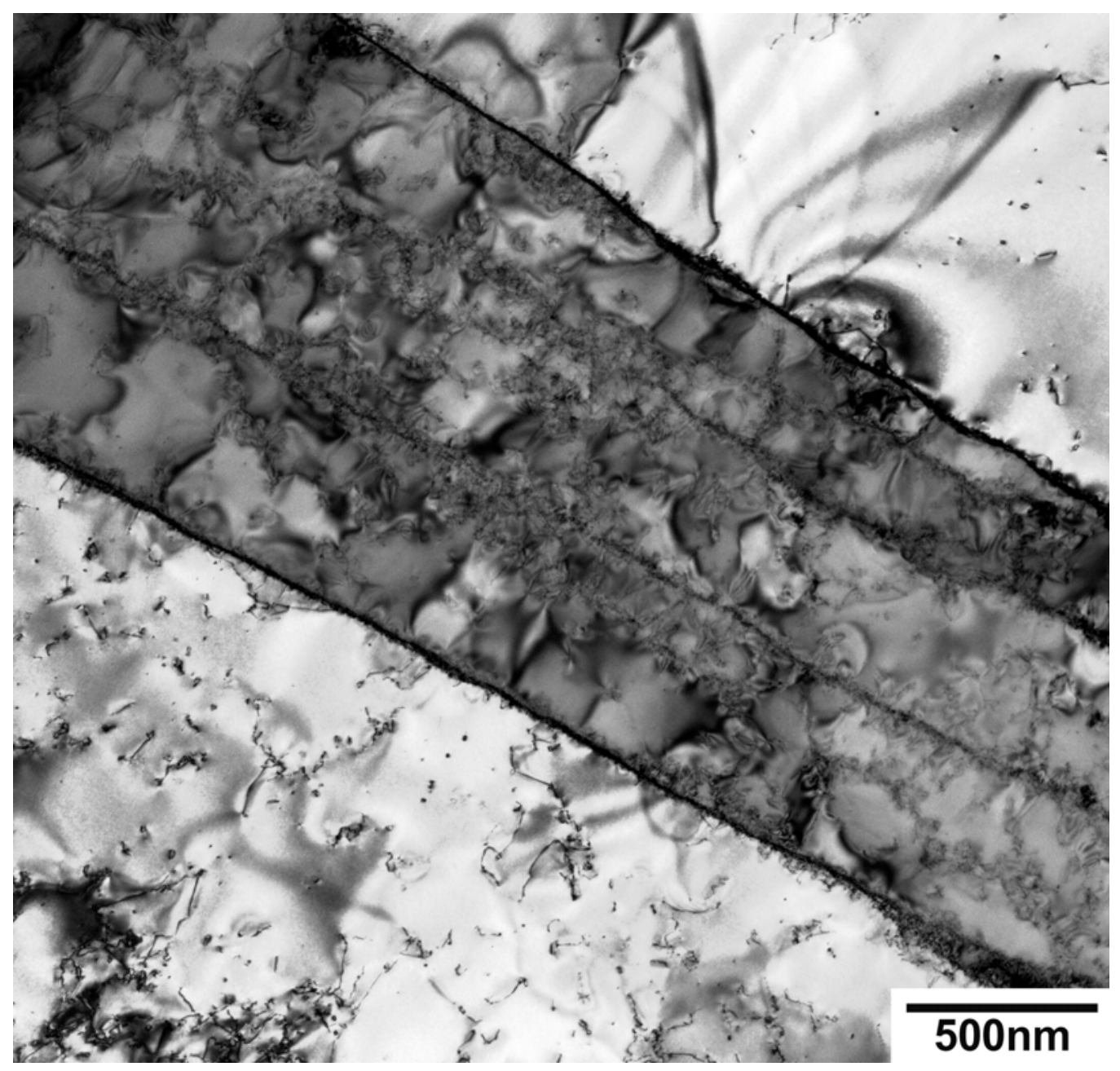

Figure 6a 


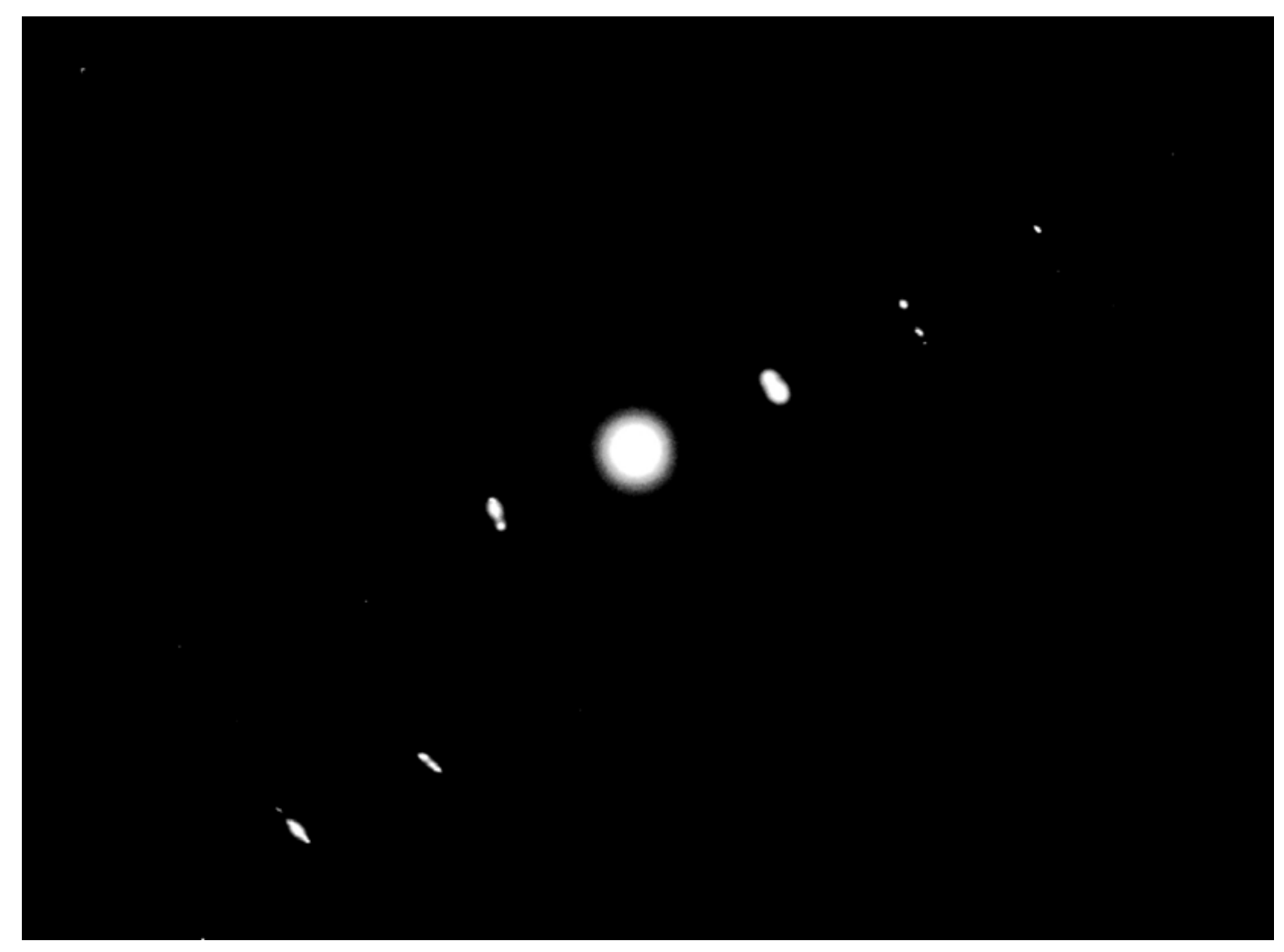

Figure 6b 


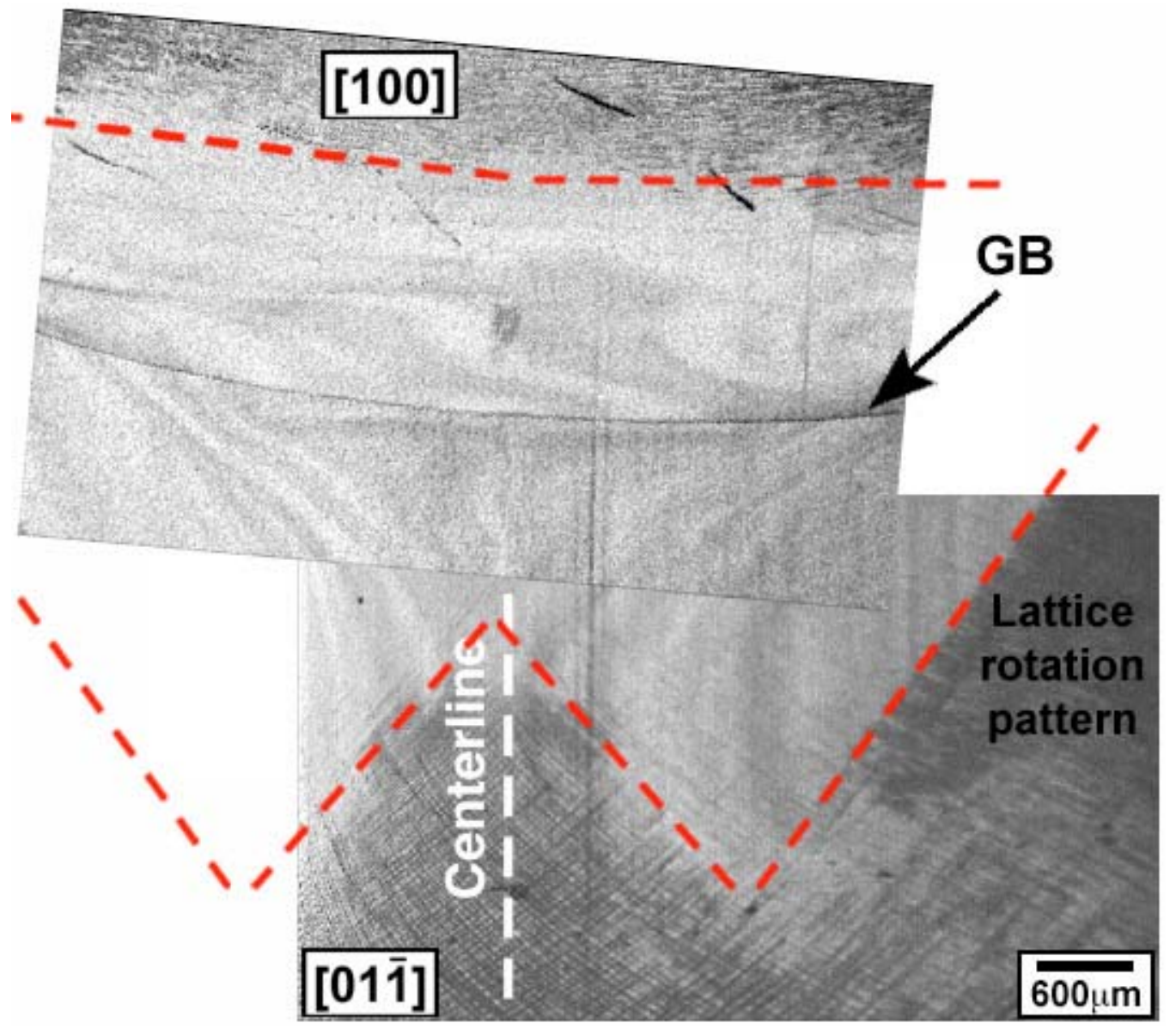

Figure 7 